\title{
Parasites promote host gene flow in a metapopulation
}

\author{
Florian Altermatt · Jürgen Hottinger · Dieter Ebert
}

Received: 20 May 2006/ Accepted: 12 October 2006/Published online: 30 November 2006

(C) Springer Science+Business Media B.V. 2006

\begin{abstract}
Local adaptation is a powerful mechanism to maintain genetic diversity in subdivided populations. It counteracts the homogenizing effect of gene flow because immigrants have an inferior fitness in the new habitat. This picture may be reversed in host populations where parasites influence the success of immigrating hosts. Here we report two experiments testing whether parasite abundance and genetic background influences the success of host migration among pools in a Daphnia magna metapopulation. In 22 natural populations of D. magna, immigrant hosts were found to be on average more successful when the resident populations experienced high prevalences of a local microsporidian parasite. We then determined whether this success is due to parasitism per se, or the genetic background of the parasites. In a common garden competition experiment, we found that parasites reduced the fitness of their local hosts relatively more than the fitness of allopatric host genotypes. Our experiments are consistent with theoretical predictions based on coevolutionary host-parasite models in metapopulations. A direct consequence of the observed mechanism is an elevated effective migration rate for the host in the metapopulation.
\end{abstract}

Keywords Immigration $\cdot$ Local parasite $\cdot$ Metapopulation $\cdot$ Coevolution $\cdot$ Daphnia magna $\cdot$ Octosporea bayeri

F. Altermatt $\cdot$ J. Hottinger $\cdot$ D. Ebert $(\bowtie)$

Zoologisches Institut, Universität Basel, Vesalgasse 1, CH-4051 Basel, Switzerland e-mail: Dieter.Ebert@unibas.ch

F. Altermatt

e-mail: Florian.Altermatt@unibas.ch

J. Hottinger

e-mail: Juergen.Hottinger@unibas.ch

F. Altermatt $\cdot$ J. Hottinger $\cdot$ D. Ebert

Département de Biologie, Unité d'Ecologie et Evolution, Université de Fribourg, Chemin du Musée 10, CH-1700 Fribourg, Switzerland

F. Altermatt $\cdot$ J. Hottinger $\cdot$ D. Ebert

Tvärminne Zoological Station, SF-10900 Hanko, Finland 


\section{Introduction}

Migration is a fundamental process for metapopulation dynamics and the persistence of local populations (Hanski et al. 1995; Gonzalez et al. 1998; Hanski 1999). It has been shown that an increased immigration rate can reduce the probability of local extinction by reinforcing local population sizes in natural and experimental systems (Hanski et al. 1995; Gonzalez et al. 1998). Migration also introduces new genetic material (Slatkin 1987), which can prevent the negative demographic consequences of inbreeding (Saccheri et al. 1998; Westemeier et al. 1998; Ebert et al. 2002; Liberg et al. 2005). These processes depend on the effective immigration rate, which is not only a function of the rate with which migrants travel among sites, but also of the fitness differences between the immigrants and the resident individuals. If immigrants experience an advantage relative to the local population members, the effective migration rate is higher than the basic migration rate, which is based on a neutral model (Ebert et al. 2002). Natural populations are often expected to be adapted to local conditions, suggesting that conspecific immigrants should be on average competitively inferior (Arnold 1977; Lively 1989; Fry 1990; De Meester et al. 2002). Thus, local adaptation counteracts the beneficial effects of migration, such as the introduction of new genetic material and the spread of favourable alleles across metapopulations (Levins 1968; Slatkin 1987; Hanski 1999).

Parasites are a ubiquitous part of the environment and have a strong influence on individual hosts as well as on entire host populations (Price 1980; Hudson and Greenman 1998; Poulin 1998; Ebert 2005). On a community level, they have also been proposed to influence host invasion success (Mitchell and Power 2003; Torchin et al. 2003) and a recent theoretical model predicts the influence of parasites on gene flow between host populations (Telschow et al. 2006). Parasites often exhibit local adaptation to their host (Lively 1989; Ebert 1994). Locally adapted parasites have been reported to have an increased fitness on a sympatric host compared to an allopatric (=immigrant) host (Lively 1989; Ebert 1994, 1998). The increased fitness on a sympatric host can be due to an increase in virulence. This means that parasites cause higher host damage on a sympatric host compared to an allopatric host (Ebert 1994). Thus, a difference in parasites' harm on local and immigrant hosts may alter the competitive abilities among the hosts. Therefore, we speculate that local parasites give immigrant hosts an advantage over resident hosts. By this, parasites could alter predictions on migration success made for single-species metapopulations (see above) by elevating the effective migration rate and thus enhance the exchange of alleles between populations and increase genetic diversity within them.

Here, we tested experimentally whether host immigration is influenced by the presence of local parasites. Therefore, we experimentally investigated the role of the microsporidian parasite Octosporea bayeri Jírovec 1936 on the migration success of its host Daphnia magna Straus 1820. D. magna occurs in the study area in Southern Finland in rockpool populations, which form a natural metapopulation; and $O$. bayeri is by far the most important parasite in this metapopulation (Ebert et al. 2001). Hosts and parasites coexist in most rockpool populations, and passive migration of resting stages allows colonization of unoccupied habitats as well as immigration into existing populations (Ranta 1979; Pajunen and Pajunen 2003). During migration, hosts might escape their native, sympatric parasite population. To understand the effect of parasites on host migration, we conducted two experiments. 
First, we tested how the parasite $O$. bayeri influences the establishment success of $D$. magna immigrants entering natural D. magna populations. These populations differed in the natural prevalence of $O$. bayeri. By using the same immigrant genotypes infected as well as uninfected we could attribute the parasite effect on host migration to the residents' parasites as well as to parasites of the immigrants. In a second experiment, we tested whether a difference in competitive abilities between hosts from two populations is a consequence of the presence of a parasite per se or due to the origin of the parasite population, being sympatric with the host or not. Therefore, we performed a competition experiment between hosts of different origin and measured their competitive success in the presence of either's local (=sympatric) parasite. We used a reciprocal common garden design, as we could then attribute the effect of parasites on host competition to the parasites' genetic background. We showed that in this metapopulation local parasite genotypes give immigrating host genotypes a competitive advantage over residents.

\section{Materials and methods}

\section{Daphnia magna and Octosporea bayeri}

The freshwater crustacean D. magna (Crustacea: Cladocera) is widely distributed along the coast of the Baltic Sea, inhabiting water-filled rockpools (Pajunen and Pajunen 2003). These pools are spatially separated and genetic differentiation between the rockpool populations is strong (Haag et al. 2005). Periods of asexual reproduction during summer are intermitted by sexual reproduction when resting eggs are produced. These resting eggs allow overwintering and also serve as dispersal stages (Ranta 1979). Rockpool D. magna populations often harbour a wide spectrum of parasites (Bengtsson and Ebert 1998; Ebert et al. 2001; Ebert 2005). The most common parasite in our study area is the microsporidium $O$. bayeri, which can be found in about $45 \%$ of all D. magna populations (Ebert et al. 2001). It is specific to D. magna, is vertically as well as horizontally transmitted and reduces host fecundity and survival (Vizoso and Ebert 2004). Hosts and parasites are able to migrate among populations, and hosts may escape the parasite by dispersal (Green 1957; Bengtsson and Ebert 1998; Ebert et al. 2001). In May 2003, we collected D. magna females, each representing a unique genotype (=clone), hatched from overwintering eggs from 24 natural rockpool populations on 14 islands near Tvärminne Zoological Station, Finland $\left(59^{\circ} 50^{\prime} \mathrm{N}, 23^{\circ} 15^{\prime} \mathrm{E}\right)$. All populations were known to have been infected with $O$. bayeri in previous years. One or two females per population were used to establish clonal isofemale lines (=asexually reproducing genotypes). They were kept in $100 \mathrm{~mL}$ artificial medium (Klüttgen et al. 1994) at room temperature and were fed with the green algae Scenedesmus obliquus. To cure hosts from possible $O$. bayeri infections, we treated all lines with the antibiotic fumagillin (Zbinden et al. 2005). Infections of $O$. bayeri can be easily seen when the host is dissected and investigated with phase-contrast microscopy (400× magnification). By this means the success of the curing process was affirmed in all genotypes. We also typed all $D$. magna for their allozyme genotypes at five different loci. These five loci were aspartate amino transferase (Aat, enzyme commission number EC 2.6.1.1), fumarate hydratase (Fum, EC 4.2.1.2), glucose-6-phosphate isomerase (Gpi, EC 5.3.1.9), 
phosphoglucomutase (Pgm, EC 5.4.2.2) and mannose-6-phosphate isomerase (Mpi, EC 5.3.1.8) (Hebert and Beaton 1993).

The process of migration in this Daphnia metapopulation can be divided into three phases: (1) physical and passive entering of the migrant (in the resting egg stage) into a resident population. (2) Asexual competition of the immigrants with the residents during the whole summer season (10-15 asexual generations). Then, only in phase (3) potential introgression of the immigrants genes is achieved by sexual reproduction. Sexual reproduction takes place to survive harsh conditions as winter, and the recombinants only hatch afterwards. A successful introgression is only achieved when the immigrant is able to compete asexually with the residents, and the number of sexually produced resting stages might be a direct function of the frequency of the immigrant in the asexual competing population. It is an intrinsic feature of the system that phase 2 is transitory, as recombination has to take place to survive winter. But the effects of the processes in that phase are long lasting. The fate of the immigrant in phase 2 can be either pure stochastic (drift model, without selection) or influenced by selection (we predict an advantage of the immigrant due to the residents' parasites). If selection in favour of the immigrants takes place, it will finally lead to a higher genetic diversity of the resident population. The long phase of asexual competition is a feature of the Daphnia system that allows to differentiate between direct effects of local parasites and effects that occur only after recombination (Ebert et al. 2002).

All D. magna individuals used for the experiments were unique genotypes newly hatched from resting eggs. Each genotype had at time of collection a frequency of $1 / n$, where $n$ is the population size where it came from ( $n$ being usually in the order of 1,000-10,000). When the genotypes used for the experiment would be frequent in their home population at the time of sampling, the effect of local parasite selection is expected to be even stronger (Lively and Dybdahl 2000).

\section{Immigration experiment design}

In this experiment, we aimed to test whether the success of a rare immigrant depends on the infection status of the immigrant itself and on the prevalence of the infection in the resident population. Immigration success was monitored with the help of genetic markers.

\section{Preparation of the immigrants}

We chose 22 D. magna genotypes (each from a different population) as immigrants from the stock that had been collected in May 2003. All of them had been treated against possible parasites as previously described and kept for another 2 weeks in the laboratory. Then we split each of the 22 D. magna genotypes into four isofemale lines. These isofemale lines were started with 2-day-old juveniles. Two of the isofemale lines per genotype were sham infected (treatment 1, Fig. 1a), while the two other lines of each genotype were infected with an isolate of their sympatric parasite O. bayeri (treatment 2, Fig. 1a). This resulted in 22 sympatric host-parasite combinations. We used 22 separate parasite isolates to infect the isofemale lines of the 22 different genotypes. The parasite isolates were made of infected $D$. magna females that had been collected at the end of May 2003 in the same 22 pools as the D. magna 
a) Experimental design of the immigration experiment

\author{
replicated resident
}

one out of 22 natural rockpool populations

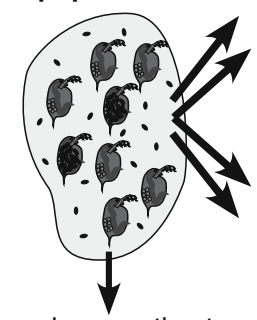

prevalence estimate

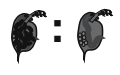

populations in buckets
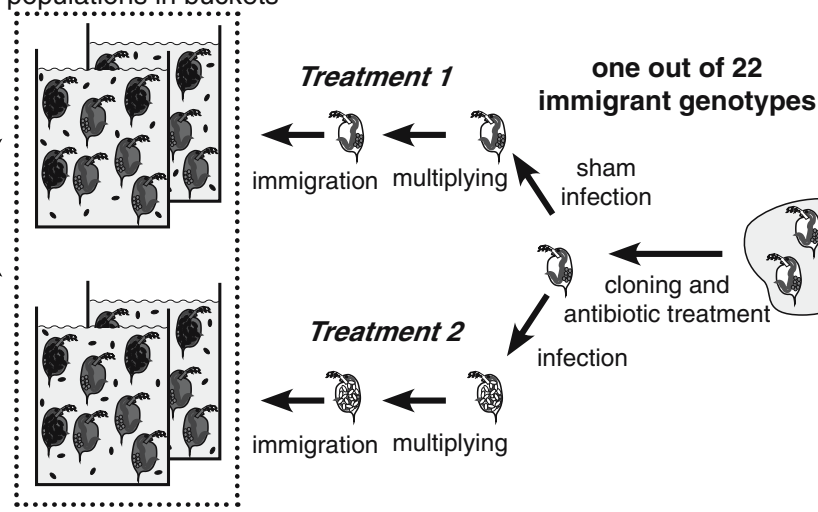

b) Experimental design of the common garden competition experiment

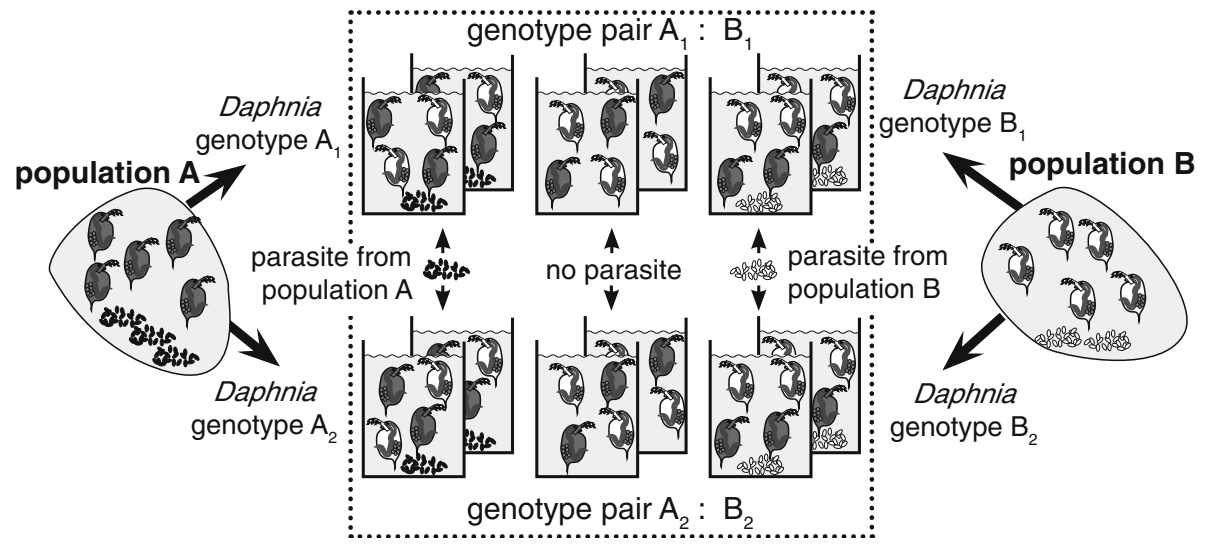

Fig. 1 a Experimental setup shown for one of 22 population replicates of the immigration experiment. The dotted line surrounds the experimental replicates, while the adjacent parts depict the setting up. Population samples of a natural rockpool population of D. magna and the corresponding natural pool water (containing parasite spores) were split on four buckets filled with water from the rockpool of origin (adjacent on the left). Prevalence of $O$. bayeri was determined. One immigrant genotype originating from a different population had been previously cloned (adjacent on the right), treated with antibiotics against $O$. bayeri and then experimentally introduced either uninfected (treatment 1 ) or infected with its sympatric parasite (treatment 2). Initial frequency of the immigrant in the bucket populations was set to $10 \%$. Relative fitness of the immigrant was monitored by following its genotype frequency during clonal competition over the experimental season. b Experimental setup shown for one out of the ten pairs of populations of the common garden competition experiment. The dotted line surrounds the experimental replicates, while the adjacent parts depict the setting up. From natural rockpool populations ( $A$ and $B$, adjacent on the left and on the right) two different $D$. magna genotypes were isolated, respectively $\left(A_{1}\right.$ and $A_{2}, B_{1}$ and $B_{2}$ ). They were treated with antibiotics against $O$. bayeri and cloned. These genotypes were competing in pairs in their asexual phase in presence of either sympatric parasite $(O$. bayeri isolates from populations $A$ and $B$, respectively) or in absence of parasites. Relative fitness of the genotypes was monitored by following their genotype frequencies over time 
genotypes. For each parasite isolate about 20 infected females had been homogenized to produce spore suspensions. About 50,000 spores per host individual were then added in each of the two isofemale lines per host genotype of treatment 2 . In the sham infection lines (treatment 1) aliquots of uninfected, homogenized $D$. magna were added. Infection success was confirmed on days 15-20 post-infection (Zbinden et al. 2005). The infection process took place in 10-100 mL artificial medium. After 10 days all lines were kept in $0.8 \mathrm{~L}$ artificial medium in the laboratory and fed moderately. To avoid maternal effects, only animals of the second generation's offspring were used as immigrants for the experiment.

\section{Preparation of the resident populations}

During immigration, one or a few individuals enter a numerical larger group of resident individuals. To create such resident populations, we used 22 natural rockpool populations from 13 different islands. All of these rockpool populations were naturally infected with $O$. bayeri, but with extensive variation in prevalence. From each of these 22 natural rockpool populations we established four resident population replicates in 10-L buckets (Fig. 1a). On the 19 and 21 July 2003, the water of each rockpool was filtered through a $125 \mu \mathrm{m}$ filter to exclude invertebrates but not parasite spores and food particles and split in four buckets per rockpool, each receiving $6 \mathrm{~L}$ of water. Then we added a random population sample of $90 \mathrm{D}$. magna (in one rockpool-replicate 45) from the corresponding natural rockpool to each of these buckets. The population sample reflected the actual epidemiological and demographic structure of the natural population. This gave a total of 88 resident populations (four resident populations from each of the 22 rockpools), which were placed on an island close to natural rockpools. Replicates were randomly arranged. At the same time, a fifth sample was taken from each pool, wherefrom we obtained a prevalence estimate by checking 50 animals for the presence of $O$. bayeri (in one of 22 natural populations a fifth sample was not available). Another 22 females per population were genotyped at the five loci mentioned above and the summed numbers of alleles present at these loci were taken as an estimate of genetic diversity for each resident population.

\section{Immigration into the resident populations}

The four isofemale lines (two infected and two uninfected) of each immigrant genotype were assigned to the four resident populations from one rockpool population. Assignment of the immigrant genotypes was arbitrary, except that the immigrants had to be genetically different for at least one of five allozyme markers from their resident populations. The mean distance between the natural rockpool of the resident population and the rockpool were the corresponding immigrant had been collected from was 2,800 m (SE $\pm 320 \mathrm{~m})$. Adult females of each genotype's isofemale lines were used as immigrants and added to the resident populations. Thus, two uninfected and two infected isofemale lines within each immigrant genotype entered resident populations of the same genetic and demographic composition (Fig. 1a). Each immigrant genotype started at a frequency of $10 \%$ in its resident population. Immigration success was monitored by scoring the frequencies of the genetic markers at which residents and immigrants differed. The experiment took place during the asexual growth season of D. magna when only clonal 
competition occurs. Thus, changes in allele frequencies are equal to changes in genotype frequencies and therefore the most comprehensive and direct measurement of relative fitness (Hartl and Clark 1997). Eight days after the release of the immigrants we sampled about $8 \%$ of the total population; another 35 days later, the entire population (population densities stayed roughly constant between these two samplings). A sub-sample of these animals was genotyped to identify the frequency of immigrants and residents. In the first sample, we genotyped on average 39.6 animals per replicate $(\mathrm{SE}=1.52)$, in the second sample we genotyped on average 58.7 animals per replicate $(\mathrm{SE}=3.25)$.

\section{Common garden competition experiment}

To test for the effect of the parasites' genetic identity on host competitive abilities, we conducted a competition experiment in a common garden. In this experiment, pairs of $D$. magna genotypes from different populations were competing against each other in the presence of either's sympatric $O$. bayeri parasite or in the absence of parasites. We measured relative fitness of each genotype in the different parasite treatments with the help of genetic markers.

\section{Preparation of the genotype pairs}

We used $D$. magna genotypes from 20 populations. The populations were assigned to ten population pairs, and these were the unit of replication. Each population contributed two genotypes. All of these genotypes had been previously collected and cured as described above. This gave a total of 40 genotypes; 18 of which had also been used in the immigration experiment. For each population pair two pairs of genotypes were established (Fig. 1b). Genotypes within a pair were distinguishable with genetic markers in at least one of five allozyme loci mentioned above. Within a pair, the two populations of origin were always located on different islands (mean distance $=2,300 \mathrm{~m}, \mathrm{SE} \pm 210 \mathrm{~m}$ ). The 40 genotypes were individually mass cultured in the laboratory in $4 \mathrm{~L}$ jars under standardized conditions.

\section{Setting up the experiment}

On the 16 and 17 July 2003, 20 adult females of each genotype per pair and replicate were released together into plastic buckets containing $6 \mathrm{~L}$ of $20 \mu \mathrm{m}$-filtered water from a rockpool free of $D$. magna and $O$. bayeri. Thus, the experiment took place in a common garden environment. The buckets were randomly arranged and placed outdoors on an island next to natural rockpools. For each of the ten population pairs, we had three treatments per pair of genotypes, two replicates with the parasite from the rockpool of one population, two with the parasite from the other rockpool population and two without parasites ( $=10$ pairs of populations $\times 2$ genotypes $\times 3$ treatments $\times 2$ replicates $=120$ buckets; Fig. 1b). The parasites were added to the buckets by placing 20 dead infected $D$. magna females collected from the corresponding rockpools (freshly killed with $\mathrm{CO}_{2}$ ). Parasite spores were passively released from the decaying cadavers of these hosts, allowing the infection to spread in the bucket population. Twenty dead females from parasite free laboratory cultures were added to each replicate of the controls. The controls are needed to allocate the parasite effect on the specific origin of the parasites and to detect potential 
contaminations. After 54 days (equivalent to about five asexual D. magna generations), we collected all animals. Population densitiy between there replicates was fairly constant. We genotyped 69-72 animals (mean $=70.5, \mathrm{SE}=0.2$ ) from each replicate to obtain genotype frequencies as a direct measurement of relative fitness of the competing genotypes. This number corresponded to about $10 \%$ of the whole replicate's population. We also checked each replicate for the presence of $O$. bayeri infections in a sample of 20 adults that were homogenized and screened for parasite spores.

Fitness measurement and analysis

An increase in frequency during the phase of asexual competition is the most direct demonstration of a genotype's advantage. To achieve comparability, we estimated the relative fitness $w$ of one genotype relative to the other (e.g. $A$ to $B$ ) or relative to the resident population for each replicate using the formula: $\ln (w)^{*} t=\ln \left(A_{t} / B_{t}\right)-$ $\ln \left(A_{0} / B_{0}\right)$ (Hartl and Clark 1997), where $A_{t}, A_{0}, B_{t}$ and $B_{0}$ are the frequencies of the genotypes at time $t$ (measured in days) and 0 . As the experiments took place during asexual reproduction of $D$. magna, only clonal competition occurred. Thus, changes in allele frequencies are equal to changes in genotype frequencies and therefore $\ln (w)$ is a comprehensive measurement of relative fitness (Hartl and Clark 1997) and was used in the statistical analysis as dependent variable. Furthermore, the $\ln (w)$ estimates, obeyed the requirement of normal distributed residuals in analysis of variance in the immigration experiment, while the frequency data did not. Relative fitness cannot be calculated for replicates in which the observed frequency of one genotype was zero. Thus, in the immigration experiment, we conservatively estimated the frequency of the extinct genotype as $1 /(n+1)$, where $n$ is the number genotyped. We excluded replicates where the experimental populations went extinct due to unknown reasons or where one genotype was fixed and less than ten individuals could be genotyped for the analysis, which led to the exclusion of eight replicates of the first and 13 replicates of the second sampling in the immigration experiment. We were interested in population level effects of parasites on host migration. This is strongly reflected in our experimental design. We used host population as the unit of replication in both experiments. The biological relevant unit of replication in the immigration experiment was "population" and in the common garden competition experiment "population-pair". Genotypes were nested within population. In the common garden competition experiment, we additionally used always two host genotypes per population and a cocktail of parasites from 20 infected hosts per population. Therefore, in both experiments the effect of local parasites holds for the level of population and not only for individual host genotypes. Statistical analyses were performed with R software (R Development Core Team 2003).

\section{Results}

Immigration success

The immigration experiment revealed that host immigration is influenced by parasites of the resident population as well as by the infection status of the immigrants. First, immigration success of uninfected hosts significantly correlated with the 
prevalence of the parasite $O$. bayeri in the replicated resident populations (Fig. 2). The prevalence in these resident populations at the time of immigration varied naturally between 6 and $100 \%$. During the entire experimental period of 43 days (equivalent to about four asexual generations of D. magna), uninfected D. magna immigrants were more successful in more heavily parasitized resident populations (Fig. 2b; Spearman rank correlation with relative fitness, $r_{\mathrm{s}}=0.70, p=0.0005$, $n=21)$. This effect was clearly visible at the second sample, but not yet significant at the first sampling after 8 days (Spearman rank correlation with relative fitness, $r_{\mathrm{s}}=0.44, p=0.06, n=22$ ). The immigration success of infected hosts did not correlate significantly with the prevalence in the resident population neither after 43 days (Spearman rank correlation with relative fitness, $r_{\mathrm{s}}=0.44, p=0.06, n=21$ ) nor after 8 days (Spearman rank correlation with relative fitness, $r_{\mathrm{s}}=0.41$, $p=0.073, n=20)$. Second, the success of immigrant hosts was influenced by their own infection status. Infected immigrants had a significantly lower relative fitness (mean relative fitness per day compared to residents $=1.20$ ) than the uninfected immigrants of the same genotype during the first 8 days of the experiment (mean relative fitness per day compared to residents $=1.40$; Table 1 , factor "infection status immigrant"). The difference between infected and uninfected immigrants, however, was no longer found for the period between days 8 and 43 (mean relative fitness per day 1.02 and 1.08, respectively; Table 2, factor "infection status immigrant"). During both time intervals there was a significant population effect on immigration success (Tables 1,2). It is important to note that the population effect includes the combined influence of the immigrant genotype, the resident's population gene pool and demography and the parasite prevalence in the resident population.
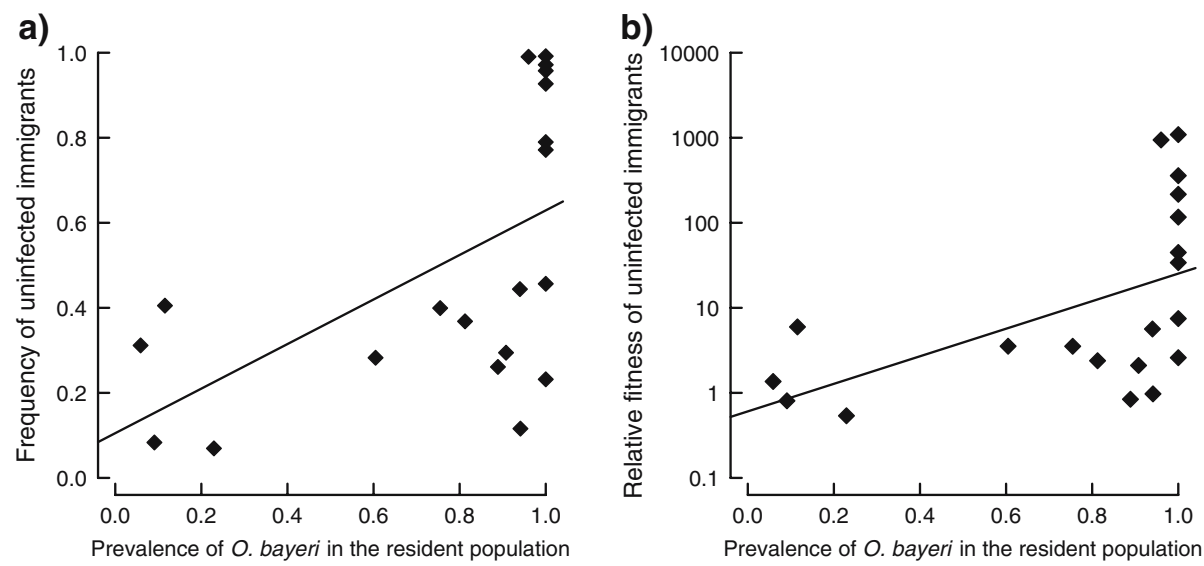

Fig. 2 Frequencies (a) and relative fitness (b) of uninfected Daphnia magna immigrant genotypes during the 43 days after immigration plotted against the prevalence of Octosporea bayeri in the resident population at the time of immigration. Immigrants are significantly more successful when they migrate into heavily infected resident populations (Spearman rank correlations: frequencies: $r_{\mathrm{s}}=0.64, p=0.002, n=21$; fitness: $\left.r_{\mathrm{s}}=0.70, p=0.0005, n=21\right)$. The line is a linear regression (a) and an exponential function (b) fitted to the data. Note that the frequency and fitness rank orders of the two graphs differ slightly, because frequencies are averaged using the arithmetic mean, while mean fitness values are geometric means (Crawley 2002) 
Table 1 Two-way ANOVA for the effects of resident population ("population") and infection status of the immigrant on its immigration success as well as their interaction

\begin{tabular}{lrlcc}
\hline Source of variation & $d f$ & MS & F & $p$ \\
\hline Population & 20 & 0.115 & 20.33 & $<0.00001$ \\
Infection status immigrant & 1 & 0.061 & 5.54 & 0.029 \\
Population $\times$ infection status & 19 & 0.011 & 1.94 & 0.04 \\
Error & 39 & 0.0056 & &
\end{tabular}

Dependent variable: fitness of the immigrants (infected or uninfected) relative to the residents during the first 8 days of the experiment. "Population" is a random, "infection status" a fixed effect. Test for normality: Shapiro-Wilk $W=0.97, p=0.08$

Further analysis revealed another factor influencing the success of immigrants. Relative fitness of the immigrants over the whole experimental period correlated negatively with the number of alleles that were present in the resident population (number of alleles summed over five loci; Spearman rank correlation, $r_{\mathrm{s}}=-0.46$, $p=0.029, n=22$ ). The prevalence of $O$. bayeri in the resident populations did not depend on their genetic diversity (Spearman rank correlation, $r_{\mathrm{s}}=-0.18, p=0.44$, $n=21)$.

\section{Common garden competition experiment}

In the reciprocal common garden experiment, we tested whether the origin of the parasite influenced the outcome of competition among host genotypes from different populations. We found significant shifts from the staring frequency of $50 \%$ caused by the presence of sympatric or allopatric parasites (Wilcoxon signed rank test using frequency data: $V=3, p=0.0098, n=10$ pairs of pools, Fig. 3a; Wilcoxon signed rank test using relative fitness values: $V=5, p=0.020, n=10$ pairs of pools, Fig. 3b). Thus, hosts were significantly inferior competitors in presence of their own, local (=sympatric) parasite compared to the presence of an allopatric parasite. A parasite screen at the end of the experiment revealed that the parasite established successfully in at least $83 \%$ of the replicates, while none of the controls became infected.

\section{Discussion}

We studied the influence of parasites on host immigration success and competition in a metapopulation of $D$. magna and its microsporidian parasite $O$. bayeri. We found

Table 2 Two-way ANOVA for the effects of resident population ("population") and infection status of the immigrant on its immigration success as well as their interaction

\begin{tabular}{lrlll}
\hline Source of variation & $d f$ & MS & F & $p$ \\
\hline Population & 20 & 0.0053 & 2.39 & 0.011 \\
Infection status immigrant & 1 & 0.0009 & 0.041 & 0.0525 \\
Population $\times$ infection status & 17 & 0.002 & 0.045 & 0.513 \\
Error & 36 & 0.002 & &
\end{tabular}

Dependent variable: fitness of the immigrants (infected or uninfected) relative to the residents between days 8 and 43 of the experiment. "Population" is a random, "infection status" a fixed effect. Test for normality: Shapiro-Wilk $W=0.97, p=0.13$ 


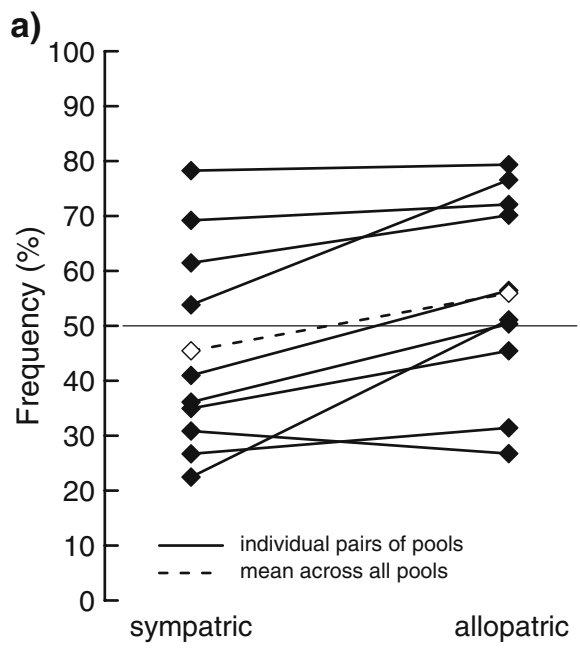

Origin of parasite

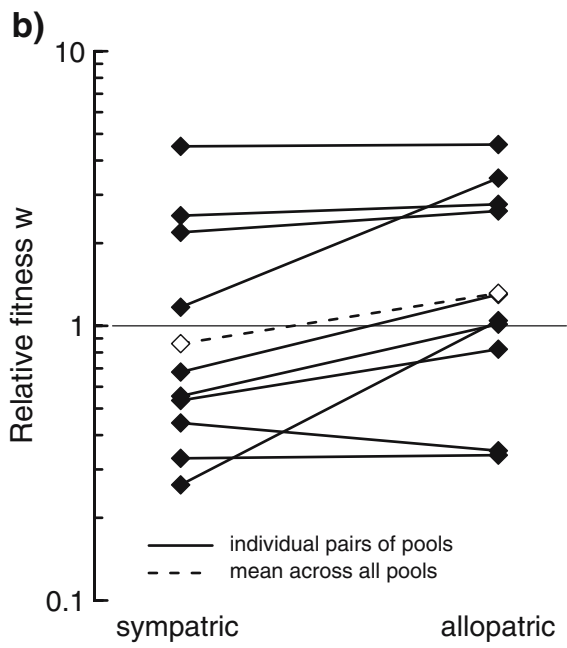

Origin of parasite

Fig. 3 Frequency (a) and relative fitness (b) of D. magna genotypes of ten pairs of populations relative to the competitors in the presence of their sympatric or the competitor's (=allopatric) parasite. Positive slopes indicate a higher competitive fitness of genotypes from one population in the presence of the allopatric parasite compared to the presence of the sympatric parasite (a Mean frequency $45 \%$ vs. $59 \%$, Wilcoxon signed rank test, $V=3, p=0.0098, n=10$ pairs; $\mathbf{b}$ mean fitness 1.31 vs. 0.86 , Wilcoxon signed rank test, $V=5, p=0.020, n=10$ pairs). Genotype frequency was measured at the end of the experiment. Starting frequency was $50 \%$ in all replicates. Fitness was estimated from genotype frequency changes across the 54 days experimental duration. Each solid point is the mean of the two frequencies or the two fitness estimates of the two genotypes, respectively. Solid lines connect pairs of populations. The dashed line with white symbols is the overall average. Note that the frequency and fitness rank orders of the two graphs differ slightly, because genotype frequencies are averaged using the arithmetic mean, while mean fitness values are geometric means (Crawley 2002)

that local parasites influence host immigration success and that the influence of parasites is due to their genetic identity. Immigrant hosts profit by the presence of local parasites and thus effective migration rate is increased. This effect of parasites had been predicted for an "all else being equal" situation, but has not been shown empirically before.

In many examples resident and migrating individuals differ in their relative fitness, whereby transience and settlement are usually costly for the migrants (Bélichon et al. 1996). We showed in our experiments that the presence of parasites in the resident population facilitated the establishment of immigrant hosts. In particular high prevalences in the resident host population promoted the success of uninfected hosts during a time period of at least four to five asexual generations. This implies that in natural systems, host immigration success may be higher during time periods when the parasite prevalence in the resident populations is high. In the field, Daphnia resting stages (=ephippia) production and prevalence of O. bayeri peak in mid summer and fall (Ranta 1979; Lass and Ebert 2006). It is currently not clear whether migration of resting eggs takes also mainly place at these times, but it is possible. Ephippia are often picked up by wind from dry sediments of shallow pools and may thus reach other pools (D. Ebert, personal observation). In our metapopulation, many pools fall dry during July just about when $O$. bayeri prevalence peaks 
(Lass and Ebert 2006). Besides the presence of a parasite in the residence population, uninfected immigrants might have profited not only because of being uninfected, but also because they encountered only allopatric parasites in the resident population.

Our analysis of immigrant success revealed a significant population effect. Part of this variation may be explained by the different environmental conditions and different demography of the source and the resident population at the time of the experiment. Another part of this variation may be explained by different levels of inbreeding in the resident populations. Rockpool D. magna populations are known to suffer from the negative effects of inbreeding (Ebert et al. 2002). Consistent with this is our finding that immigrants were more successful in resident populations with low-allelic diversity, which can be used as an estimate for inbreeding (Haag et al. 2005). Differences in habitat qualities and the genetic compositions of the populations are part of this metapopulation system. Our finding of a significant parasite effect across a naturally broad range of these factors emphasizes its strength.

We also found that uninfected host immigrants had a higher relative fitness than the same immigrant genotypes being infected. This is not surprising and consistent with comparative studies on invasive species (Mitchell and Power 2003; Torchin et al. 2003). This advantage of being a parasite free immigrant was however only significant during the initial phase after immigration. We speculate that as time moved on, both the initial uninfected and infected immigrant genotypes became infected with the residents' local parasites, blurring their initial difference in infection status. Still, the initial advantage of uninfected migrants compared to infected migrants could make the difference between a successful early establishment of the migrant or not.

A number of parasites, including some parasites of D. magna, have been shown to be more virulent on their sympatric host than on allopatric hosts (Lively 1989; Ebert 1994, 1998). Therefore, we hypothesized that the success of immigrants not only depends on the presence of parasites in the host population per se, but might be influenced by their origin: local parasite adaptation may promote the establishment of foreign host immigrants (Gandon et al. 1996) by harming local hosts more than the immigrants. There are some studies that dealt with parasite-related expression of immune traits and effects of dispersal (Kurtz et al. 2002; Møller et al. 2004), but they did not manipulate parasites themselves. We experimentally manipulated both host and parasite populations. We did not test for local adaptation of the parasite itself (Kawecki and Ebert 2004), but instead tested for a corollary of it, namely host competitive fitness. It is important to use host competitive fitness, rather than some trait, which is inversely proportional to fitness in hosts and parasites (like resistance), as host fitness is not necessarily equal to the inverse of parasite fitness (Gower and Webster 2005). We predicted a reduced fitness of the local hosts relative to allopatric hosts in presence of the former sympatric parasite. In a common garden competition experiment, we found support for this hypothesis. On average, the fitness of a genotype, relative to its competitor, was significantly higher in the presence of the competitor's sympatric parasite. This is the first time that this effect has been shown empirically. It has been predicted by theoretical models that the gradient of resistance (=local adaptation) is not an asymptotic characteristic but an average distribution over time (Gandon et al. 1996). Thus even some inversed gradients (in our experiment 1 of 10) are expected. For other parasites of $D$. magna local adaptation has been demonstrated (Ebert 1994, 1998) and therefore it is a likely explanation for 
the negative effect of local parasites on their host's competitive abilities in this study as well. The D. magna-O. bayeri-system is ideal to study these effects of local adaptation. Although Daphnia have a cyclic parthenogenetic life cycle, the results can be representative for a wider range of organisms. First, there are other important taxonomic groups having a very similar live cycle, living in metapopulation systems and having parasites, as for example aphids (Weisser 2000). Second, as we only studied the effects during the asexual phase of the daphnids live cycle, the conclusions could be applied to other asexual host organisms where local adaptation has been studied (Lively 1989; Lively et al. 2004). In both cases, local parasites could give immigrating hosts an advantage relative to resident hosts. More precisely it is not only parasites in general, but sympatric parasites shifting the competitive outcome of resident and immigrant hosts in favour of the immigrants. By this, parasites alter predictions on migration success made for single-species metapopulations by elevating the effective migration rate (Arnold 1977; De Meester et al. 2002).

In the common garden competition experiment, all competing D. magna were uninfected at the beginning of the experiment and parasites were added only in form of dead infected hosts. Infective spores were taken up by the competing hosts equivalent to the natural process. The observed effect may therefore be a consequence of differential infectivity and/or by expressing different levels of virulence in those hosts that became infected. We cannot distinguish between these two hypotheses. But a distinction is not relevant for the conclusion of our study, as under natural conditions a parasite's effect on a certain host genotype is among others influenced by both infectivity and virulence.

Acknowledgements We thank H. Ganz, T. Kawecki, M. Kölliker, S. Lass, M. Zbinden, T. Zumbrunn, S. Zweizig and the anonymous reviewers for comments to earlier versions of the manuscript. The study was supported by the Swiss National Science Foundation. F.A. thanks the Tomcsik-Foundation and the Swiss Academy of Sciences for financial support during the fieldwork. This is part of project nr 97524006 at Tvärminne Zoological Station.

\section{References}

Arnold SJ (1977) Polymorphism and geographic variation in feeding-behavior of garter snake Thamnophis elegans. Science 197:676-678

Bengtsson J, Ebert D (1998) Distribution and impacts of microparasites on Daphnia in a rockpool metapopulation. Oecologia (Berlin) 115:213-221

Bélichon S, Clobert J, Massot M (1996) Are there differences in fitness components between philopatric and dispersing individuals? Acta Ecologica 17:503-517

Crawley MJ (2002) Statistical computing. An introduction to data analysis using S-Plus. Wiley, Chichester

De Meester L, Gomez A, Okamura B, Schwenk K (2002) The monopolization hypothesis and the dispersal-gene flow paradox in aquatic organisms. Acta Oecol-Int J Ecol 23:121-135

Ebert D (1994) Virulence and local adaptation of a horizontally transmitted parasite. Science 265:1084-1086

Ebert D (1998) Experimental evolution of parasites. Science 282:1432-1435

Ebert D (2005) Ecology, epidemiology, and evolution of parasitism in Daphnia. National Library of Medicine (US), National Center for Biotechnology Information, Bethesda, MD

Ebert D, Haag C, Kirkpatrick M, Riek M, Hottinger JW, Pajunen VI (2002) A selective advantage to immigrant genes in a Daphnia metapopulation. Science 295:485-488

Ebert D, Hottinger JW, Pajunen VI (2001) Temporal and spatial dynamics of parasites in a Daphnia metapopulation: which factors explain parasite richness? Ecology 82:3417-3434 
Fry JD (1990) Trade-off in fitness on different hosts: evidence from a selection experiment with a pythophagous mite. Am Nat 136:569-580

Gandon S, Capowiez Y, Dubois Y, Michalakis Y, Olivieri I (1996) Local adaptation and gene-forgene coevolution in a metapopulation model. Proc R Soc Lond Ser B-Biol Sci 263:1003-1009

Gonzalez A, Lawton JH, Gilbert FS, Blackburn TM, Evans-Freke I (1998) Metapopulation dynamics, abundance, and distribution in a microecosystem. Science 281:2045-2047

Gower CM, Webster JP (2005) Intraspecific competition and the evolution of virulence in a parasitic trematode. Evolution 59:544-553

Green J (1957) Parasites and epibionts of Cladocera in rockpools of Tvärminne archipelago. Arch Soc Zool Bot Fenn Vanamo 12:5-12

Haag CR, Riek M, Hottinger JW, Pajunen VI, Ebert D (2005) Genetic diversity and genetic differentiation in Daphnia metapopulations with subpopulations of known age. Genetics 170:18091820

Hanski I (1999) Metapopulation ecology. Oxford University Press, Oxford

Hanski I, Pöyry J, Pakkala T, Kuussaari M (1995) Multiple equilibira in metapopulation dynamics. Nature 377:618-621

Hartl DL, Clark AG (1997) Principles of population genetics, 3rd edn. Sinauer Associates, Sunderland

Hebert PDN, Beaton MJ (1993) Methodologies for allozyme analysis using cellulose acetate electrophoresis, 2nd edn. Helena Laboratories, Beaumont, TX

Hudson P, Greenman J (1998) Competition mediated by parasites: biological and theoretical progress. Trends Ecol Evol 13:387-390

Kawecki TJ, Ebert D (2004) Conceptual issues in local adaptation. Ecol Lett 7:1225-1241

Klüttgen B, Dülmer U, Engels M, Ratte HT (1994) ADaM, an artificial freshwater for the culture of zooplankton. Water Res 28:743-746

Kurtz J, Klappert K, Schneider W, Reinhold K (2002) Immune defence, dispersal and local adaptation. Evol Ecol Res 4:431-439

Lass S, Ebert D (2006) Apparent seasonality of parasite dynamics: analysis of cyclic prevalence patterns. Proc R Soc B-Biol Sci 273:199-206

Levins R (1968) Evolution in changing environments. Princeton University Press, Princeton, NJ

Liberg O, et al (2005) Severe inbreeding depression in a wild wolf (Canis lupus) population. Biol Lett 1:17-20

Lively C, Dybdahl ME, Jokela J, Osnas EE, Delph LE (2004) Host sex and local adaptation by parasites in a snail-trematode interaction. Am Nat 164(Suppl):6-18

Lively CM (1989) Adaptation by a parasitic trematode to local-populations of its snail host. Evolution 43:1663-1671

Lively CM, Dybdahl MF (2000) Parasite adaptation to locally common host genotypes. Nature 405:679-681

Mitchell CE, Power AG (2003) Release of invasive plants from fungal and viral pathogens. Nature 421:625-627

Møller AP, Martin-Vivaldi M, Soler JJ (2004) Parasitism, host immune defence and dispersal. J Evol Biol 17:603-612

Pajunen VI, Pajunen I (2003) Long-term dynamics in rockpool Daphnia metapopulations. Ecography 26:731-738

Poulin R (1998) Evolutionary ecology of parasites: from individuals to communities. Chapman \& Hall, London

Price PW (1980) Evolutionary biology of parasites. Princeton University Press, Princeton, NJ

R Development Core Team (2003) R: a language and environment for statistical computing, version 1.6.2. R Foundation for Statistical Computing, Vienna, Austria

Ranta E (1979) Niche of Daphnia species in rockpools. Arch Hydrobiol 87:205-223

Saccheri I, Kuusaari M, Kankare M, Vikman P, Fortelius W, Hanski I (1998) Inbreeding and extinction in a butterfly metapopulation. Nature 392:392-395

Slatkin M (1987) Gene flow and the geographic structure of natural populations. Science 236:787792

Telschow A, Engelstädter J, Yamamura N, Hammerstein P, Hurst GDD (2006) Asymmetric gene flow and constraints on adaptation caused by sex ratio distorters. J Evol Biol 9:869-878

Torchin ME, Lafferty KD, Dobson AP, McKenzie VJ, Kuris AM (2003) Introduced species and their missing parasites. Nature 421:628-630

Vizoso DB, Ebert D (2004) Within-host dynamics of a microsporidium with horizontal and vertical transmission: Octosporea bayeri in Daphnia magna. Parasitology 128:31-38 
Weisser WW (2000) Metapopulation dynamics in an aphid-parasitoid system. Entomol Exp Appl 97:83-92

Westemeier RL et al (1998) Tracking the long-term decline and recovery of an isolated population. Science 282:1695-1698

Zbinden M, Lass S, Refardt D, Hottinger J, Ebert D (2005) Octosporea bayeri: fumidil B inhibits vertical transmission in Daphnia magna. Exp Parasitol 109:58-61 